\title{
Approximate Analytic Solutions of Time-Fractional Hirota-Satsuma Coupled KdV Equation and Coupled MKdV Equation
}

\author{
Jincun Liu and Hong Li \\ School of Mathematical Sciences, Inner Mongolia University, 235 West Daxue Road, Hohhot 010021, China \\ Correspondence should be addressed to Jincun Liu; ljincun@163.com
}

Received 29 October 2012; Accepted 28 January 2013

Academic Editor: Abdel-Maksoud A. Soliman

Copyright (C) 2013 J. Liu and H. Li. This is an open access article distributed under the Creative Commons Attribution License, which permits unrestricted use, distribution, and reproduction in any medium, provided the original work is properly cited.

By introducing the fractional derivative in the sense of Caputo and combining the pretreatment technique to deal with long nonlinear items, the generalized two-dimensional differential transform method is proposed for solving the time-fractional Hirota-Satsuma coupled KdV equation and coupled MKdV equation. The presented method is a numerical method based on the generalized Taylor series expansion which constructs an analytical solution in the form of a polynomial. The numerical results show that the generalized two-dimensional differential transform method is very effective for the fractional coupled equations.

\section{Introduction}

In the last past decade, the fractional differential equations have been widely used in various fields of physics and engineering. The analytical approximation of such problems has attracted great attention and became a considbased onerable interest in mathematical physics. Some powerful methods including the homotopy perturbation method [1], Adomian decomposition method $[2,3]$, variational iteration method $[4,5]$, homotopy analysis method $[6,7]$, fractional complex transform method [8], and generalized differential transform method [9] have been developed to obtain exact and approximate analytic solutions. These solution techniques are more clear and realistic methods for fractional differential equations, because they give the approximate solutions of the considered problems without any linearization or discretization.

The variational iteration method and the homotopy perturbation method were first proposed by Professor He in $[10,11]$, respectively. The idea of the variational iteration method is to construct correction functionals using general Lagrange multipliers identified optimally via the variational theory, and the initial approximations can be freely chosen with unknown constants. Recently, Wu and Lee proposed a fractional variational iteration method for fractional differ- ential equation based on the modified Riemann CLiouville derivative, which is more effective to solve fractional differential equation [12]. This method has been developed by many authors, see [13-16] and the references cited therein. The homotopy perturbation method, which does not require a small parameter in an equation, has a significant advantage that it provides an analytical approximate solution to a wide range of nonlinear problems in applied sciences. Recently, the fractional complex transform is developed to convert the fractional differential equation to its differential partner and gave a geometrical explanation [8]. These methods are more effective for solving the linear and nonlinear fractional differential equations.

The differential transform method was used firstly by Zhou in 1986 to study electric circuits [17]. The differential transform is an iterative procedure based on the Taylor series expansion which constructs an analytic solution in the form of a polynomial. The method is well addressed in [18-23]. Recently, the generalized differential transform method is developed for obtaining approximate analytic solutions for some linear and nonlinear differential equations of fractional order $[24,25]$.

The aim of this paper is to directly extend the generalized two-dimensional differential transform method to 
obtain the approximate analytic solutions of a time-fractional Hirota-Satsuma coupled KdV equation,

$$
\begin{gathered}
D_{t}^{\alpha} u=\frac{1}{2} u_{x x x}-3 u u_{x}+3 v w_{x}+3 v_{x} w, \\
D_{t}^{\alpha} v=-v_{x x x}+3 u v_{x}, \\
D_{t}^{\alpha} w=-w_{x x x}+3 u w_{x}, \quad t>0,0<\alpha \leq 1,
\end{gathered}
$$

and a time-fractional coupled $\mathrm{MKdV}$ equation,

$$
\begin{gathered}
D_{t}^{\beta} u=\frac{1}{2} u_{x x x}-3 u^{2} u_{x}+\frac{3}{2} v v_{x}+3 u v_{x}+3 u_{x} v-3 \lambda u_{x} \\
D_{t}^{\beta} v=-v_{x x x}-3 v v_{x}-3 u_{x} v_{x}+3 u^{2} v_{x}+3 \lambda v_{x} \\
t>0,0<\beta \leq 1
\end{gathered}
$$

where $\lambda$ is a constant and $\alpha$ and $\beta$ are parameters describing the order of the time-fractional derivatives of $u(x, t), v(x, t)$, and $w(x, t)$, respectively. The fractional derivatives are considered in the Caputo sense. In the case of $\alpha=1$ and $\beta=1$, (1) and (2) reduce to the classical Hirota-Satsuma coupled KdV equation and coupled MKdV equation [26], respectively.

The Caputo fractional derivative is considered here because it allows traditional initial and boundary conditions to be included in the formulation of the problem.

Definition 1 (see [27]). The fractional derivative of $f(x)$ in the Caputo sense is defined as

$$
\begin{aligned}
D^{\nu} f(x) & =J_{0}^{m-v} D^{m} f(x) \\
& =\frac{1}{\Gamma(m-v)} \int_{0}^{x}(x-t)^{m-\nu-1} f^{(m)}(t) d t,
\end{aligned}
$$

for $m-1<v<m$ and $m \in N, x>0$. Here, $J_{0}^{\mu}$ is the Riemann-Liouville integral operator of order $\mu>0$, defined by

$$
J_{0}^{\mu} f(x)=\frac{1}{\Gamma(\mu)} \int_{0}^{x}(x-t)^{\mu-1} f(t) d t, \quad \mu>0 .
$$

Definition 2 (see [28]). For $m$ to be the smallest integer that exceeds $\alpha$, the Caputo time-fractional derivative of order $\alpha>$ 0 is defined as

$$
D_{t}^{\alpha} u(x, t)=\frac{\partial^{\alpha} u}{\partial t^{\alpha}}= \begin{cases}\frac{1}{\Gamma(m-\alpha)} \\ \times \int_{0}^{t}(t-\xi)^{m-\alpha-1} & \\ \times \frac{\partial^{m} u(x, \xi)}{\partial \xi^{m}} d \xi, & \text { for } m-1<\alpha<m, \\ \frac{\partial^{m} u(x, t)}{\partial t^{m}}, & \text { for } \alpha=m \in N,\end{cases}
$$

and the space-fractional derivatives of Caputo type can be defined analogously.
Lemma 3 (see [29]). The Caputo fractional derivative of the power function satisfies

$$
D_{t}^{\alpha} t^{p}= \begin{cases}\frac{\Gamma(p+1)}{\Gamma(p-\alpha+1)} t^{p-\alpha}, & n-1<\alpha<n, \\ 0, & p>n-1, p \in \mathbb{R}, \\ & n-1<\alpha<n, \\ & p \leq n-1, p \in \mathbb{N} .\end{cases}
$$

\section{Generalized Two-Dimensional Differential Transform Method (GDTM)}

Consider a function of two variables $u(x, t)$ and suppose that it can be represented as a product of two single variable functions, that is, $u(x, t)=f(x) g(t)$. Based on the properties of generalized two-dimensional differential transform, the function $u(x, t)$ can be represented as

$$
\begin{aligned}
u(x, t) & =\sum_{k=0}^{\infty} F_{\alpha}(k)\left(x-x_{0}\right)^{k \alpha} \sum_{h=0}^{\infty} G_{\beta}(h)\left(t-t_{0}\right)^{h \beta} \\
& =\sum_{k=0}^{\infty} \sum_{h=0}^{\infty} U_{\alpha, \beta}(k, h)\left(x-x_{0}\right)^{k \alpha}\left(t-t_{0}\right)^{h \beta}
\end{aligned}
$$

where $0<\alpha, \beta \leq 1, U_{\alpha, \beta}(k, h)=F_{\alpha}(k) G_{\beta}(h)$ is called the spectrum of $u(x, t)$. The generalized two-dimensional differential transform of the function $u(x, t)$ is given by

$$
\begin{aligned}
U_{\alpha, \beta}(k, h)= & \frac{1}{\Gamma(\alpha k+1) \Gamma(\beta h+1)} \\
& \times\left[\left(D_{x}^{\alpha}\right)^{k}\left(D_{t}^{\beta}\right)^{h} u(x, t)\right]_{\left(x_{0}, t_{0}\right)},
\end{aligned}
$$

where $\left(D_{x}^{\alpha}\right)^{k}=D_{x}^{\alpha} D_{x}^{\alpha} \cdots D_{x}^{\alpha}, k$ is times. In the case of $\alpha=$ 1 and $\beta=1$, the generalized two-dimensional differential transform (8) reduces to two-dimensional differential transform.

The fundamental theorems of the generalized twodimensional differential transform are as follows.

Theorem 4. Suppose that $U_{\alpha, \beta}(k, h), V_{\alpha, \beta}(k, h)$, and $W_{\alpha, \beta}(k, h)$ are the differential transformations of the functions $u(x, t)$, $v(x, t)$, and $w(x, t)$, respectively:

(a) if $u(x, t)=v(x, t) \pm w(x, t)$, then $U_{\alpha, \beta}(k, h)=V_{\alpha, \beta}$ $(k, h) \pm W_{\alpha, \beta}(k, h)$.

(b) if $u(x, t)=a v(x, t), a \in \mathbb{R}$, then $U_{\alpha, \beta}(k, h)=a V_{\alpha, \beta}(k$, h).

(c) if $u(x, t)=v(x, t) w(x, t)$, then $U_{\alpha, \beta}(k, h)=$ $\sum_{r=0}^{k} \sum_{s=0}^{h} V_{\alpha, \beta}(r, h-s) W_{\alpha, \beta}(k-r, s)$.

(d) if $u(x, t)=\left(x-x_{0}\right)^{n \alpha}\left(t-t_{0}\right)^{m \beta}$, then $U_{\alpha, \beta}(k, h)=\delta(k-$ $n) \delta(h-m)$.

(e) if $u(x, t)=D_{x}^{\alpha} v(x, t), 0<\alpha \leq 1$, then $U_{\alpha, \beta}(k, h)=$ $(\Gamma(\alpha(k+1)+1) / \Gamma(\alpha k+1)) V_{\alpha, \beta}(k+1, h)$. 
Theorem 5. If $u(x, t)=f(x) g(t)$, function $f(x)=x^{\lambda} h(x)$, where $\lambda>-1$ and $h(x)$ has the generalized Taylor series expansion $h(x)=\sum_{n=0}^{\infty} a_{n}\left(x-x_{0}\right)^{\alpha n}$,

(i) $\beta<\lambda+1$ and $\alpha$ arbitrary, or

(ii) $\beta \geq \lambda+1, \alpha$ arbitrary, and $a_{n}=0$ for $n=0,1, \ldots, m-$ 1 , where $m-1<\beta \leq m$,

then the generalized differential transform (8) becomes

$$
U_{\alpha, \beta}(k, h)=\frac{1}{\Gamma(\alpha k+1) \Gamma(\beta h+1)}\left[D_{x_{0}}^{\alpha k}\left(D_{t_{0}}^{\beta}\right)^{h} u(x, t)\right]_{\left(x_{0}, t_{0}\right)} .
$$

If $u(x, t)=D_{x_{0}}^{\gamma} v(x, t), m-1<\gamma \leq m$, and $v(x, t)=f(x)$ $g(t)$, then

$$
U_{\alpha, \beta}(k, h)=\frac{\Gamma(\alpha k+\gamma+1)}{\Gamma(\alpha k+1)} V_{\alpha, \beta}\left(k+\frac{\gamma}{\alpha}, h\right) .
$$

Some details of the aformentioned theorems can be found in [30].

\section{Applications of GDTM}

3.1. Fractional Hirota-Satsuma Coupled KdV Equation. Consider the following time-fractional Hirota-Satsuma coupled KdV equation:

$$
\begin{gathered}
D_{t}^{\alpha} u=\frac{1}{2} u_{x x x}-3 u u_{x}+3(v w)_{x}, \\
D_{t}^{\alpha} v=-v_{x x x}+3 u v_{x}, \\
D_{t}^{\alpha} w=-w_{x x x}+3 u w_{x}, \quad t>0,0<\alpha \leq 1,
\end{gathered}
$$

subject to the initial conditions

$$
\begin{gathered}
u(x, 0)=\frac{1}{3}\left(\beta-8 \gamma^{2}\right)+4 \gamma^{2} \tanh ^{2}(\gamma x), \\
v(x, 0)=\frac{-4\left(3 \gamma^{4} c_{0}-2 \beta \gamma^{2} c_{2}+4 \gamma^{4} c_{2}\right)}{3 c_{2}^{2}}+\frac{4 \gamma^{2}}{c_{2}} \tanh ^{2}(\gamma x), \\
w(x, 0)=c_{0}+c_{2} \tanh ^{2}(\gamma x),
\end{gathered}
$$

where $c_{0}, \mathcal{c}_{2}, \beta$, and $\gamma$ are arbitrary constants.

The exact solutions of (11) and (12), for the special case of $\alpha=1$, given in [26], are

$$
\begin{gathered}
u(x, t)=\frac{1}{3}\left(\beta-8 \gamma^{2}\right)+4 \gamma^{2} \tanh ^{2}[\gamma(x+\beta t)], \\
v(x, t)=\frac{-4\left(3 \gamma^{4} c_{0}-2 \beta \gamma^{2} c_{2}+4 \gamma^{4} c_{2}\right)}{3 c_{2}^{2}} \\
+\frac{4 \gamma^{2}}{c_{2}} \tanh ^{2}[\gamma(x+\beta t)], \\
w(x, t)=c_{0}+c_{2} \tanh ^{2}[\gamma(x+\beta t)] .
\end{gathered}
$$

Suppose that the solutions $u(x, t), v(x, t)$, and $w(x, t)$ can be represented as the products of single-valued functions, respectively. Applying the generalized two-dimensional differential transform to both sides of (11) and using the related theorems, we have

$$
\begin{aligned}
\frac{\Gamma(\alpha(h+1)+1)}{\Gamma(\alpha h+1)} U_{1, \alpha}(k, h+1) & \\
= & \frac{1}{2}(k+1)(k+2)(k+3) U_{1, \alpha}(k+3, h) \\
& -3 \sum_{r=0}^{k} \sum_{s=0}^{h}(k+1-r) U_{1, \alpha}(r, h-s) U_{1, \alpha}(k+1-r, s) \\
& +3(k+1) \sum_{r=0}^{k+1} \sum_{s=0}^{h} V_{1, \alpha}(r, h-s) W_{1, \alpha}(k+1-r, s), \\
\frac{\Gamma(\alpha(h+1)+1)}{\Gamma(\alpha h+1)} V_{1, \alpha}(k, h+1) & -(k+1)(k+2)(k+3) V_{1, \alpha}(k+3, h) \\
& -3 \sum_{r=0}^{k} \sum_{s=0}^{h}(k+1-r) U_{1, \alpha}(r, h-s) V_{1, \alpha}(k+1-r, s), \\
\left.\frac{\Gamma(\alpha(h+1)+1)}{\Gamma(\alpha h}+1\right) & W_{1, \alpha}(k, h+1) \\
= & -(k+1)(k+2)(k+3) W_{1, \alpha}(k+3, h) \\
& -3 \sum_{r=0}^{k} \sum_{s=0}^{h}(k+1-r) U_{1, \alpha}(r, h-s) W_{1, \alpha}(k+1-r, s) .
\end{aligned}
$$

The generalized two-dimensional differential transforms of the initial conditions can be obtained as follows:

$$
\begin{gathered}
U_{1, \alpha}(k, 0)=V_{1, \alpha}(k, 0)=W_{1, \alpha}(k, 0)=0 \\
\text { if } k=1,3,5, \ldots, \\
U_{1, \alpha}(0,0)=\frac{1}{3}\left(\beta-8 \gamma^{2}\right), \quad U_{1, \alpha}(2,0)=4 \gamma^{4}, \\
U_{1, \alpha}(4,0)=-\frac{8}{3} \gamma^{6}, \quad U_{1, \alpha}(6,0)=\frac{68}{45} \gamma^{8}, \ldots, \\
V_{1, \alpha}(0,0)=-\frac{4\left(3 \gamma^{4} c_{0}-2 \beta \gamma^{2} c_{2}+4 \gamma^{4} c_{2}\right)}{3 c_{2}^{2}}, \\
V_{1, \alpha}(2,0)=\frac{4 \gamma^{4}}{c_{2}}, \\
V_{1, \alpha}(4,0)=-\frac{8 \gamma^{6}}{3 c_{2}}, \quad V_{1, \alpha}(6,0)=\frac{68 \gamma^{8}}{45 c_{2}}, \ldots, \\
W_{1, \alpha}(0,0)=c_{0}, \quad W_{1, \alpha}(2,0)=c_{2} \gamma^{2}, \\
W_{1, \alpha}(4,0)=-\frac{2 c_{2}}{3} \gamma^{4}, \quad W_{1, \alpha}(6,0)=\frac{17 c_{2}}{45} \gamma^{6}, \ldots
\end{gathered}
$$


Utilizing the recurrence relations (14) and the transformed initial conditions, we can obtain all the $U_{1, \alpha}(k, h)$, $V_{1, \alpha}(k, h)$, and $W_{1, \alpha}(k, h)$ with the help of Mathematica. Moreover, substituting all $U_{1, \alpha}(k, h)$ into (7), we obtain the series form solution

$$
u(x, t)=u_{0}(x, t)+u_{1}(x, t)+u_{2}(x, t)+\cdots,
$$

where

$$
\begin{aligned}
& u_{0}(x, t) \\
& =\frac{1}{3}\left(\beta-8 \gamma^{2}\right)+4 \gamma^{4} x^{2}-\frac{8 \gamma^{6}}{3} x^{4}+\frac{68 \gamma^{8}}{45} x^{6}-\frac{248 \gamma^{10}}{315} x^{8}+\cdots, \\
& u_{1}(x, t) \\
& =\left\{\frac{8 \gamma^{4}\left(c_{2} \beta-3 c_{0}\left(-1+\gamma^{2}\right)\right)}{c_{2}} x\right. \\
& +\frac{16 \gamma^{6}\left(c_{2}\left(9-2 \beta-9 \gamma^{2}\right)+6 c_{0}\left(-1+\gamma^{2}\right)\right)}{3 c_{2}} x^{3} \\
& +\frac{8 \gamma^{8}\left(-51 c_{0}\left(-1+\gamma^{2}\right)+c_{2}\left(17 \beta+180\left(-1+\gamma^{2}\right)\right)\right)}{15 c_{2}} x^{5} \\
& +\cdots\} \frac{t^{\alpha}}{\Gamma(1+\alpha)} \\
& u_{2}(x, t) \\
& =\left\{\left(\frac{8 \gamma^{4}\left(-12 c_{0} \gamma^{2}\left(-1+\gamma^{2}\right)+c_{2}\left(\beta^{2}-18 \gamma^{2}\left(-1+\gamma^{2}\right)\right)\right)}{c_{2}}\right.\right. \\
& +\frac{16 \gamma^{6}\left(51 c_{0} \gamma^{2}\left(-1+\gamma^{2}\right)-2 c_{2}\left(\beta^{2}-54 \gamma^{2}\left(-1+\gamma^{2}\right)\right)\right)}{c_{2}} x^{2} \\
& -\frac{8 \gamma^{8}\left(744 c_{0} \gamma^{2}\left(-1+\gamma^{2}\right)+c_{2}\left(-17 \beta^{2}+2016 \gamma^{2}\left(-1+\gamma^{2}\right)\right)\right)}{3 c_{2}} x^{4} \\
& +\cdots)\} \frac{t^{2 \alpha}}{\Gamma(1+2 \alpha)}, \ldots
\end{aligned}
$$

The closed forms of $u_{0}(x, t), u_{1}(x, t)$, and $u_{2}(x, t)$ are

$$
\begin{aligned}
u_{0}(x, t)= & \frac{1}{3}\left(\beta-8 \gamma^{2}\right)+4 \gamma^{2} \tanh ^{2}(\gamma x) \\
u_{1}(x, t)= & \frac{\gamma^{3} \sec ^{2}(\gamma x)}{c_{2}} \\
& \times\left[24 c_{0}+8 c_{2} \beta-24 c_{0} \gamma^{2}+48 c_{2}\left(\gamma^{2}-1\right) \tanh ^{2}(\gamma x)\right] \\
& \times \frac{\tanh (\gamma x) t^{\alpha}}{\Gamma(1+\alpha)},
\end{aligned}
$$

$$
\begin{aligned}
u_{2}(x, t)= & \frac{\gamma^{6}\left(1-\gamma^{2}\right)}{c_{2}} \\
& \times\left\{96\left(c_{0}+2 c_{2}\right) \sec ^{2}(\gamma x)\right. \\
& +\frac{8 c_{2} \beta^{2}}{\gamma^{2}\left(1-\gamma^{2}\right)}(2-\cosh (2 \gamma x)) \sec ^{4}(\gamma x) \\
& +6\left[15 c_{0}+94 c_{2}-88 c_{2} \cosh (2 \gamma x)\right. \\
& \times \frac{t^{2 \alpha}}{\Gamma(1+2 \alpha)} .
\end{aligned}
$$

Similarly, substituting all $V_{1, \alpha}(k, h)$ and all $W_{1, \alpha}(k, h)$ into (7), respectively, we obtain the series form solutions

$$
\begin{gathered}
v(x, t)=v_{0}(x, t)+v_{1}(x, t)+v_{2}(x, t)+\cdots, \\
w(x, t)=w_{0}(x, t)+w_{1}(x, t)+w_{2}(x, t)+\cdots .
\end{gathered}
$$

The closed forms of $v_{0}(x, t), v_{1}(x, t)$ and $v_{2}(x, t)$ are

$$
\begin{gathered}
v_{0}(x, t)=\frac{-4\left(3 \gamma^{4} c_{0}-2 \beta \gamma^{2} c_{2}+4 \gamma^{4} c_{2}\right)}{3 c_{2}^{2}}+\frac{4 \gamma^{2}}{c_{2}} \tanh ^{2}(\gamma x), \\
v_{1}(x, t)=\frac{8 c_{0} \beta \gamma^{3} \sec ^{2}(\gamma x) \tanh (\gamma x)}{c_{2}} \frac{t^{\alpha}}{\Gamma(1+\alpha)}, \\
v_{2}(x, t) \\
=\left[\frac{8 \beta^{2} \gamma^{4}\left(3 \sec ^{2}(\gamma x)-2\right)}{c_{2} \cosh ^{2}(\gamma x)}\right. \\
\left.\quad+\frac{576 \gamma^{6}\left(1-\gamma^{2}\right)\left(c_{0}+2 c_{2} \tanh ^{2}(\gamma x)\right) \tanh ^{2}(\gamma x)}{c_{2}^{2} \cosh ^{4}(\gamma x)}\right] \\
\times \frac{t^{2 \alpha}}{\Gamma(1+2 \alpha)} .
\end{gathered}
$$

The closed forms of $w_{0}(x, t), w_{1}(x, t)$, and $w_{2}(x, t)$ are

$$
\begin{gathered}
w_{0}(x, t)=c_{0}+c_{2} \tanh ^{2}(\gamma x), \\
w_{1}(x, t)=\frac{2 c_{2} \beta \gamma \sec ^{2}(\gamma x) \tanh (\gamma x)}{c_{2}} \frac{t^{\alpha}}{\Gamma(1+\alpha)}, \\
w_{2}(x, t)=\left[2 c_{2} \beta^{2} \gamma^{2} \sec ^{2}(\gamma x)\left(3 \sec ^{2}(\gamma x)-2\right)\right. \\
+144\left(c_{0}+2 c_{2}\right) \gamma^{4}\left(1-\gamma^{2}\right) \\
\times \sec ^{4}(\gamma x) \tanh ^{2}(\gamma x)-288 c_{2} \gamma^{4}\left(1-\gamma^{2}\right) \\
\left.\times \sec ^{6}(\gamma x) \tanh ^{2}(\gamma x)\right] \frac{t^{2 \alpha}}{\Gamma(1+2 \alpha)} .
\end{gathered}
$$




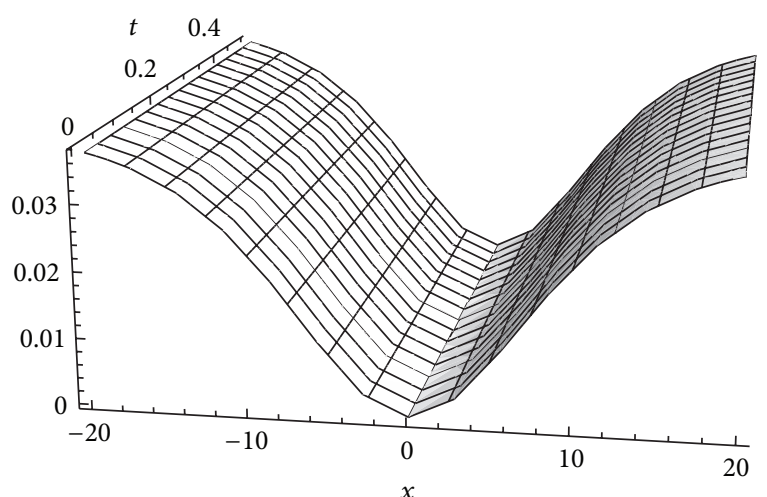

(a)

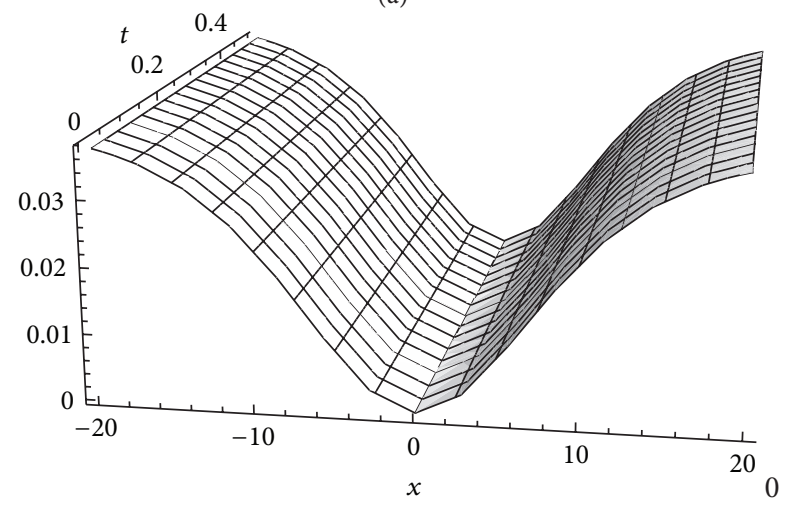

(b)

FIGURE 1: The surface shows the solution $u(x, t)$ of (11): (a) exact solution; (b) approximate solution of (22) when $\alpha=c_{0}=c_{2}=1$, $\gamma=0.1$, and $\beta=0.08$.

The approximate solutions of (11) in finite series forms are given by

$$
\begin{gathered}
u(x, t)=u_{0}(x, t)+u_{1}(x, t)+u_{2}(x, t), \\
v(x, t)=v_{0}(x, t)+v_{1}(x, t)+v_{2}(x, t), \\
w(x, t)=w_{0}(x, t)+w_{1}(x, t)+w_{2}(x, t) .
\end{gathered}
$$

In order to verify whether the approximate solutions of (22)-(24) lead to higher accuracy, we draw the figures of the approximate solutions of (22)-(24) with $\alpha=1$, as well as the exact solutions (13) when $c_{0}=c_{2}=1, \gamma=0.1$, and $\beta=$ 0.08. It can be seen from Figures 1(a) to 3(b) that the solutions obtained by the presented method is nearly identical with the exact solutions. So, we conclude that a good approximation is achieved by using the GDTM.

In the following, we will construct an approximate solution of (11) with the new initial conditions,

$$
\begin{gathered}
u(x, 0)=\frac{\beta-2 \gamma^{2}}{3}+2 \gamma^{2} \tanh ^{2}(\gamma x), \\
v(x, 0)=-\frac{4 \gamma^{2} c_{0}\left(\beta+\gamma^{2}\right)}{3 c_{1}^{2}}+\frac{4 \gamma^{2}\left(\beta+\gamma^{2}\right)}{3 c_{1}} \tanh (\gamma x), \\
w(x, 0)=c_{0}+c_{1} \tanh (\gamma x),
\end{gathered}
$$

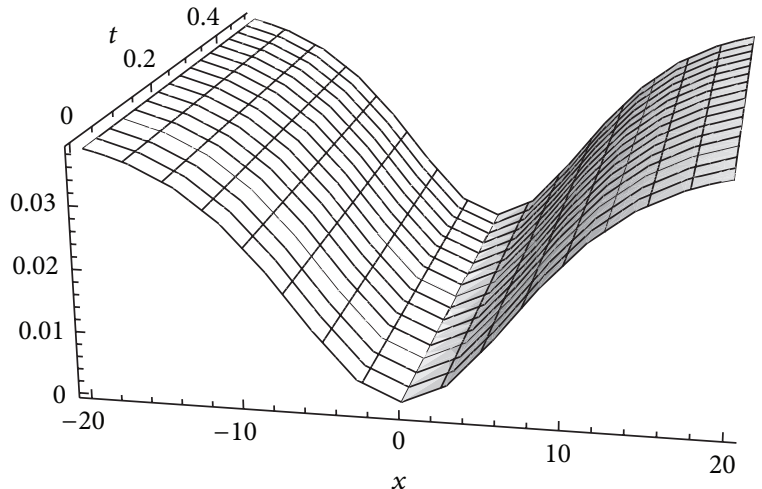

(a)

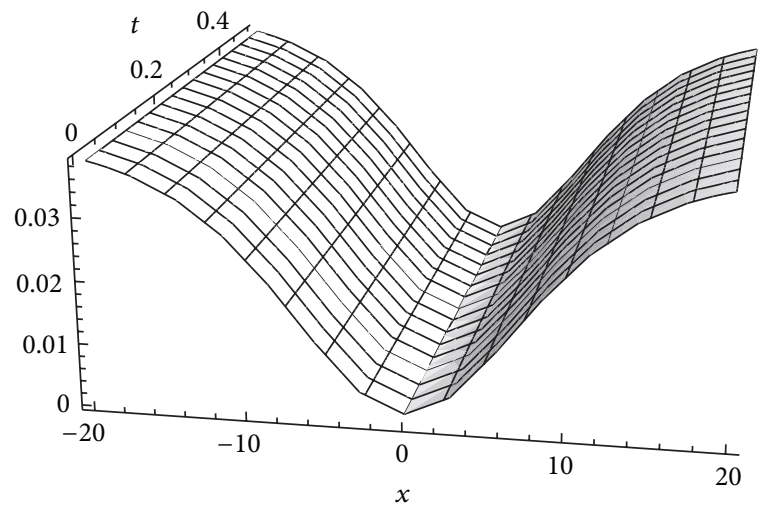

(b)

FIgURE 2: The surface shows the solution $v(x, t)$ of (11): (a) exact solution; (b) approximate solution of (23) when $\alpha=c_{0}=c_{2}=1$, $\gamma=0.1$, and $\beta=0.08$.

The generalized two-dimensional differential transforms of the initial conditions of (25) are given by

$$
\begin{gathered}
U_{1, \alpha}(k, 0)=0, \quad \text { if } k=1,3,5, \ldots, \\
V_{1, \alpha}(k, 0)=W_{1, \alpha}(k, 0)=0 \quad \text { if } k=2,4,6, \ldots, \\
U_{1, \alpha}(0,0)=\frac{1}{3}\left(\beta-2 \gamma^{2}\right), \quad U_{1, \alpha}(2,0)=2 \gamma^{4}, \\
U_{1, \alpha}(4,0)=-\frac{4}{3} \gamma^{6}, \quad U_{1, \alpha}(6,0)=\frac{34}{45} \gamma^{8}, \ldots, \\
V_{1, \alpha}(0,0)=-\frac{4 c_{0} \gamma^{2}\left(\beta+\gamma^{2}\right)}{3 c_{1}^{2}}, \\
V_{1, \alpha}(1,0)=\frac{4 \gamma^{3}\left(\beta+\gamma^{2}\right)}{3 c_{1}}, \\
V_{1, \alpha}(3,0)=-\frac{4 \gamma^{5}\left(\beta+\gamma^{2}\right)}{9 c_{1}}, \ldots, \\
W_{1, \alpha}(0,0)=c_{0}, \quad W_{1, \alpha}(1,0)=c_{1} \gamma, \\
W_{1, \alpha}(3,0)=-\frac{c_{1}}{3} \gamma^{3}, \quad W_{1, \alpha}(5,0)=\frac{2 c_{1}}{15} \gamma^{5}, \ldots
\end{gathered}
$$


Utilizing the recurrence relations in (14) and the transformed initial conditions, we can obtain the following approximate solutions:

$$
\begin{array}{r}
u(x, t)=u_{0}(x, t)+u_{1}(x, t)+u_{2}(x, t)+u_{3}(x, t), \\
v(x, t)=v_{0}(x, t)+v_{1}(x, t)+v_{2}(x, t)+v_{3}(x, t), \\
w(x, t)=w_{0}(x, t)+w_{1}(x, t)+w_{2}(x, t)+w_{3}(x, t),
\end{array}
$$

where the closed forms of $u_{i}(x, t), v_{i}(x, t)$, and $w_{i}(x, t)(i=$ $0,1,2,3)$ are

$$
\begin{aligned}
& u_{0}(x, t)=\frac{\beta-2 \gamma^{2}}{3}+2 \gamma^{2} \tanh ^{2}(\gamma x), \\
& u_{1}(x, t)=4 \beta \gamma^{3} \operatorname{sech}^{2}(\gamma x) \tanh (\gamma x) \frac{t^{\alpha}}{\Gamma(1+\alpha)}, \\
& u_{2}(x, t)=-4 \beta^{2} \gamma^{4}(\cosh (2 \gamma x)-2) \operatorname{sech}^{4}(\gamma x) \frac{t^{2 \alpha}}{\Gamma(1+2 \alpha)} \text {, } \\
& u_{3}(x, t)=\left\{2 \gamma^{2} \operatorname{sech}^{2}(\gamma x) \tanh (\gamma x)\right. \\
& \times\left(64 \beta^{2} \gamma^{5} \operatorname{sech}^{2}(\gamma x)+144 \beta^{2} \gamma^{5} \tanh ^{4}(\gamma x)\right) \\
& -2 \gamma^{5}\left(144 \beta^{2} \gamma^{2}-8 \beta^{3}\right) \operatorname{sech}^{2}(\gamma x) \tanh ^{3}(\gamma x) \\
& -\frac{1}{88} \beta^{2} \gamma^{5}\left(878 \beta+3626 \gamma^{2}\right. \\
& +\left(179 \beta+2921 \gamma^{2}\right) \cosh (2 \gamma x) \\
& +\left(386 \beta-922 \gamma^{2}\right) \cosh (4 \gamma x) \\
& \left.+7\left(-5 \beta+\gamma^{2}\right) \cosh (6 \gamma x)\right) \\
& \times \operatorname{sech}^{8}(\gamma x) \tanh (\gamma x) \\
& \left.\times \frac{\Gamma(1+2 \alpha)}{\Gamma^{2}(1+\alpha)}\right\} \frac{t^{3 \alpha}}{\Gamma(1+3 \alpha)}, \\
& v_{0}(x, t)=-\frac{4 \gamma^{2} c_{0}\left(\beta+\gamma^{2}\right)}{3 c_{1}^{2}}+\frac{4 \gamma^{2}\left(\beta+\gamma^{2}\right)}{3 c_{1}} \tanh (\gamma x) \text {, } \\
& v_{1}(x, t)=\frac{4 \beta \gamma^{3}\left(\beta+\gamma^{2}\right) \operatorname{sech}^{2}(\gamma x)}{3 c_{1}} \frac{t^{\alpha}}{\Gamma(1+\alpha)}, \\
& v_{2}(x, t)=-\frac{8 \beta^{2} \gamma^{4}\left(\beta+\gamma^{2}\right) \operatorname{sech}^{2}(\gamma x) \tanh (\gamma x)}{3 c_{1}} \frac{t^{2 \alpha}}{\Gamma(1+2 \alpha)}, \\
& v_{3}(x, t)=\left\{\frac{\beta^{2} \gamma^{5}}{30 c_{1}}\left(\beta+\gamma^{2}\right)\right. \\
& \times\left(40\left(8 \gamma^{2}-\beta\right)-15\left(3 \beta+56 \gamma^{2}\right) \cosh (2 \gamma x)\right. \\
& +576 \gamma^{2} \cosh (4 \gamma x) \\
& \left.+\left(5 \beta-56 \gamma^{2}\right) \cosh (6 \gamma x)\right) \operatorname{sech}^{8}(\gamma x) \\
& -\left(16 \beta^{2} \gamma^{6}\left(\beta+\gamma^{2}\right)\right.
\end{aligned}
$$

$\left.\times(5+\cosh (2 \gamma x)) \operatorname{sech}^{4}(\gamma x) \tanh ^{3}(\gamma x)\right)$

$$
\begin{gathered}
\left.\times\left(3 c_{1}\right)^{-1} \frac{\Gamma(1+2 \alpha)}{\Gamma^{2}(1+\alpha)}\right\} \frac{t^{3 \alpha}}{\Gamma(1+3 \alpha)}, \\
w_{0}(x, t)=c_{0}+c_{1} \tanh (\gamma x), \\
w_{1}(x, t)=c_{1} \beta \gamma \operatorname{sech}^{2}(\gamma x) \frac{t^{\alpha}}{\Gamma(1+\alpha)},
\end{gathered}
$$$$
w_{2}(x, t)=-2 c_{1} \beta^{2} \gamma^{2} \operatorname{sech}^{2}(\gamma x) \tanh (\gamma x) \frac{t^{2 \alpha}}{\Gamma(1+2 \alpha)} \text {, }
$$$$
w_{3}(x, t)=\left\{\frac { c _ { 1 } \beta ^ { 2 } \gamma ^ { 3 } } { 4 0 } \left(40\left(8 \gamma^{2}-\beta\right)\right.\right.
$$$$
-15\left(3 \beta+56 \gamma^{2}\right) \cosh (2 \gamma x)
$$$$
+576 \gamma^{2} \cosh (4 \gamma x)
$$$$
\left.+\left(5 \beta-56 \gamma^{2}\right) \cosh (6 \gamma x)\right)
$$$$
\times \operatorname{sech}^{8}(\gamma x)+\frac{2 c_{1} \beta^{2}}{5} \gamma^{5}
$$$$
\times(7 \cosh (4 \gamma x)-9-58 \cosh (2 \gamma x))
$$$$
\left.\times \operatorname{sech}^{6}(\gamma x) \tanh ^{2}(\gamma x) \frac{\Gamma(1+2 \alpha)}{\Gamma^{2}(1+\alpha)}\right\}
$$$$
\times \frac{t^{3 \alpha}}{\Gamma(1+3 \alpha)} \text {. }
$$

3.2. Fractional Coupled $M K d V$ Equation. Consider the following time-fractional coupled MKdV equation:

$$
\begin{aligned}
D_{t}^{\beta} u=\frac{1}{2} u_{x x x}- & 3 u^{2} u_{x}+\frac{3}{2} v_{x x}+3 u v_{x}+3 u_{x} v-3 \lambda u_{x} \\
D_{t}^{\beta} v= & -v_{x x x}-3 v v_{x}-3 u_{x} v_{x}+3 u^{2} v_{x} \\
& +3 \lambda v_{x}, \quad t>0,0<\beta \leq 1
\end{aligned}
$$

subject to the initial conditions

$$
\begin{gathered}
u(x, 0)=\gamma \tanh (\gamma x), \\
v(x, 0)=\frac{1}{2}\left(4 \gamma^{2}+\lambda\right)-2 \gamma^{2} \tanh ^{2}(\gamma x),
\end{gathered}
$$

where $\gamma$ is an arbitrary constant.

The exact solutions of (29) and (30), for the special case of $\beta=1$, given in [26], are

$$
\begin{gathered}
u(x, t)=\gamma \tanh (\gamma \xi), \quad \xi=x-\left(\gamma^{2}+\frac{3}{2} \lambda\right) t \\
v(x, t)=\frac{1}{2}\left(4 \gamma^{2}+\lambda\right)-2 \gamma^{2} \tanh ^{2}(\gamma \xi) .
\end{gathered}
$$




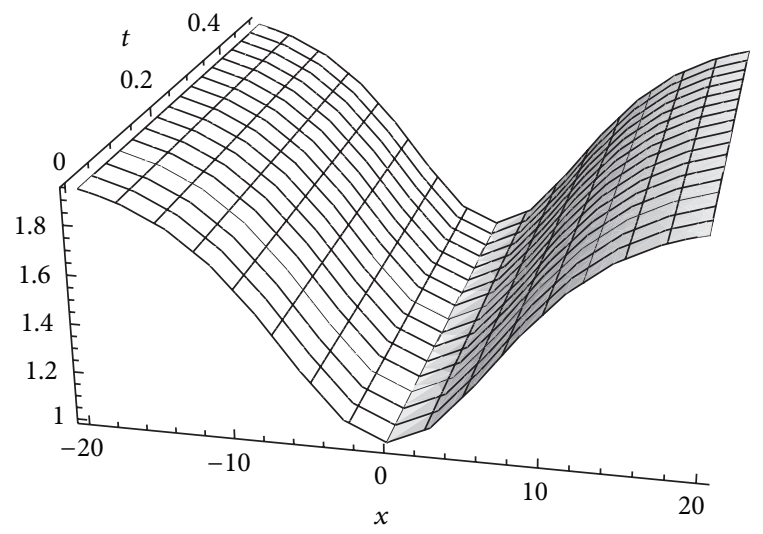

(a)

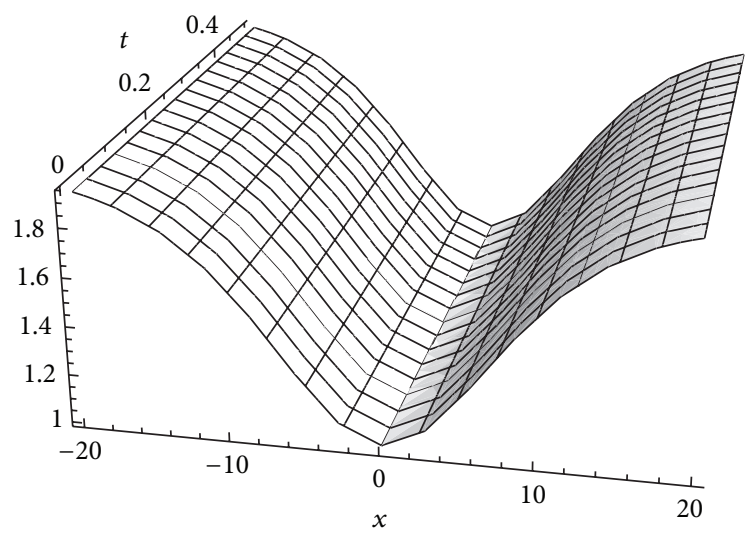

(b)

FIGURE 3: The surface shows the solution $w(x, t)$ of (11): (a) exact solution; (b) approximate solution (24) when $\alpha=c_{0}=c_{2}=1, \gamma=$ 0.1 and $\beta=0.08$.

Equation system of (29) is more complex. In order to obtain an explicit iteration scheme, we follow the pretreatment technique introduced recently by Chang $[20,21]$ to deal with long nonlinear items. Firstly, we suppose that

$$
f=v_{x}, \quad g=u_{x}, \quad p=g+3 v, \quad q=u^{2}-v+\lambda .
$$

According to (32), (29) can be equivalently written as the following form:

$$
\begin{gathered}
f=v_{x}, \quad g=u_{x}, \\
p=g+3 v, \quad q=u^{2}-v+\lambda, \\
D_{t}^{\beta} u=\frac{1}{2} p_{x x}-3 q g+3 u f, \\
D_{t}^{\beta} v=-g p_{x}+3 q f-f_{x x}+g g_{x} .
\end{gathered}
$$

Suppose that the solutions $u(x, t)$ and $v(x, t)$ can be represented as the products of single-valued functions, respectively. Applying the generalized two-dimensional differential transform to both sides of (33) and using the related theorems, we have

$$
\begin{aligned}
& F_{1, \beta}(k, h)=(k+1) V_{1, \beta}(k+1, h), \\
& G_{1, \beta}(k, h)=(k+1) U_{1, \beta}(k+1, h), \\
& P_{1, \beta}(k, h)=G_{1, \beta}(k, h)+3 V_{1, \beta}(k, h), \\
& Q_{1, \beta}(0,0)=U_{1, \beta}^{2}(0,0)-V_{1, \beta}(0,0)+\lambda, \\
& Q_{1, \beta}(k, h)=\sum_{r=0}^{k} \sum_{s=0}^{h} U_{1, \beta}(r, h-s) U_{1, \beta}(k-r, s) \\
& -V_{1, \beta}(k, h), \quad k^{2}+h^{2} \neq 0, \\
& \frac{\Gamma(\beta(h+1)+1)}{\Gamma(\beta h+1)} U_{1, \beta}(k, h+1) \\
& =\frac{1}{2}(k+1)(k+2) P_{1, \beta}(k+2, h) \\
& -3 \sum_{r=0}^{k} \sum_{s=0}^{h} Q_{1, \beta}(r, h-s) G_{1, \beta}(k-r, s) \\
& +3 \sum_{r=0}^{k+1} \sum_{s=0}^{h} U_{1, \beta}(r, h-s) F_{1, \beta}(k-r, s), \\
& \frac{\Gamma(\beta(h+1)+1)}{\Gamma(\beta h+1)} V_{1, \beta}(k, h+1) \\
& =-\sum_{r=0}^{k} \sum_{s=0}^{h}(k+1-r) G_{1, \beta}(r, h-s) P_{1, \beta} \\
& \times(k+1-r, s)-(k+1)(k+2) F_{1, \beta}(k+2, h) \\
& +3 \sum_{r=0}^{k} \sum_{s=0}^{h} Q_{1, \beta}(r, h-s) F_{1, \beta}(k-r, s) \\
& +\sum_{r=0}^{k} \sum_{s=0}^{h}(k+1-r) G_{1, \beta}(r, h-s) G_{1, \beta} \\
& \times(k+1-r, s) .
\end{aligned}
$$

Herein $F_{1, \beta}(k, h), G_{1, \beta}(k, h), P_{1, \beta}(k, h)$, and $Q_{1, \beta}(k, h)$ denote the differential transformations of the functions $f(x, t)$, $g(x, t), p(x, t)$, and $q(x, t)$, respectively.

The generalized two-dimensional differential transforms of the initial conditions of (30) can be obtained as follows:

$$
\begin{gathered}
U_{1, \beta}(k, 0)=0, \quad \text { if } k=0,2,4, \ldots, \\
V_{1, \beta}(k, 0)=0, \quad \text { if } k=1,3,5, \ldots, \\
U_{1, \beta}(1,0)=\gamma^{2}, \quad U_{1, \beta}(3,0)=-\frac{\gamma^{4}}{3}, \\
U_{1, \beta}(5,0)=\frac{2 \gamma^{6}}{15}, \quad U_{1, \beta}(7,0)=-\frac{17 \gamma^{8}}{315}, \ldots, \\
V_{1, \beta}(0,0)=\frac{1}{2}\left(4 \gamma^{2}+\lambda\right), \quad V_{1, \beta}(2,0)=-2 \gamma^{4}, \\
V_{1, \beta}(4,0)=\frac{4 \gamma^{6}}{3}, \quad V_{1, \beta}(6,0)=-\frac{34 \gamma^{8}}{45}, \ldots
\end{gathered}
$$


Utilizing the recurrence relations of (34) and the transformed initial conditions, we can obtain all the $U_{1, \beta}(k, h)$ and $V_{1, \beta}(k, h)$ with the help of Mathematica. Through the complex calculation which is similar to the solving process in Section 3.1, we have the approximate solutions of (29) in finite series,

$$
\begin{gathered}
u(x, t)=u_{0}(x, t)+u_{1}(x, t)+u_{2}(x, t)+u_{3}(x, t), \\
v(x, t)=v_{0}(x, t)+v_{1}(x, t)+v_{2}(x, t)+v_{3}(x, t),
\end{gathered}
$$

where the closed forms of $u_{i}(x, t)$ and $v_{i}(x, t)(i=0,1,2,3)$ are given by

$$
\begin{aligned}
& u_{0}(x, t)=\gamma \tanh (\gamma x), \\
& u_{1}(x, t)=-\frac{1}{2} \gamma^{2}\left(2 \gamma^{2}+3 \lambda\right) \frac{\sec ^{2}(\gamma x) t^{\alpha}}{\Gamma(1+\alpha)}, \\
& u_{2}(x, t)=-\frac{1}{2} \gamma^{3} \sec ^{2}(\gamma x) \\
& \times\left[\left(2 \gamma^{2}+3 \lambda\right)^{2}-144 \gamma^{2} \lambda \sec ^{2}(\gamma x)\right] \\
& \times \frac{\tanh (\gamma x) t^{2 \alpha}}{\Gamma(1+2 \alpha)}, \\
& u_{3}(x, t)=\left\{\frac{1}{4} \gamma^{4} \sec ^{2}(\gamma x)\right. \\
& \times\left[-2\left(2 \gamma^{2}+3 \lambda\right)^{3}+3 \sec ^{2}(\gamma x)\right. \\
& \times\left(-152 \gamma^{6}+1092 \gamma^{4} \lambda+270 \gamma^{2} \lambda^{2}+27 \lambda^{3}\right. \\
& +\left(200 \gamma^{6}-4776 \gamma^{4} \lambda-270 \gamma^{2} \lambda^{2}\right) \\
& \times \sec ^{2}(\gamma x) \\
& \left.\left.\left.+4032 \gamma^{4} \lambda \sec ^{4}(\gamma x)\right)\right]\right\} \frac{t^{3 \alpha}}{\Gamma(1+3 \alpha)} \\
& +\frac{3}{4} \gamma^{6}\left(10 \gamma^{2}-9 \lambda\right)\left(2 \gamma^{2}+3 \lambda\right) \\
& \times[2 \cosh (2 \gamma x)-3] \\
& \times \frac{\sec ^{6}(\gamma x) \Gamma(1+2 \alpha) t^{3 \alpha}}{\Gamma(1+\alpha)^{2} \Gamma(1+3 \alpha)}, \\
& v_{0}(x, t)=\frac{1}{2}\left(4 \gamma^{2}+\lambda\right)-2 \gamma^{2} \tanh ^{2}(\gamma x) \text {, } \\
& v_{1}(x, t)=2 \gamma^{3}\left(2 \gamma^{2}-3 \lambda\right) \sec ^{2}(\gamma x) \frac{\tanh (\gamma x) t^{\alpha}}{\Gamma(1+\alpha)}, \\
& v_{2}(x, t)=\left\{2\left(2 \gamma^{2}-3 \lambda\right)^{2}-3 \sec ^{2}(\gamma x)\right. \\
& \left.\times\left[4 \gamma^{4}-60 \gamma^{2} \lambda+9 \lambda^{2}+48 \gamma^{2} \lambda \sec ^{2}(\gamma x)\right]\right\} \\
& \times \frac{\gamma^{4} \sec ^{2}(\gamma x) t^{2 \alpha}}{\Gamma(1+2 \alpha)},
\end{aligned}
$$

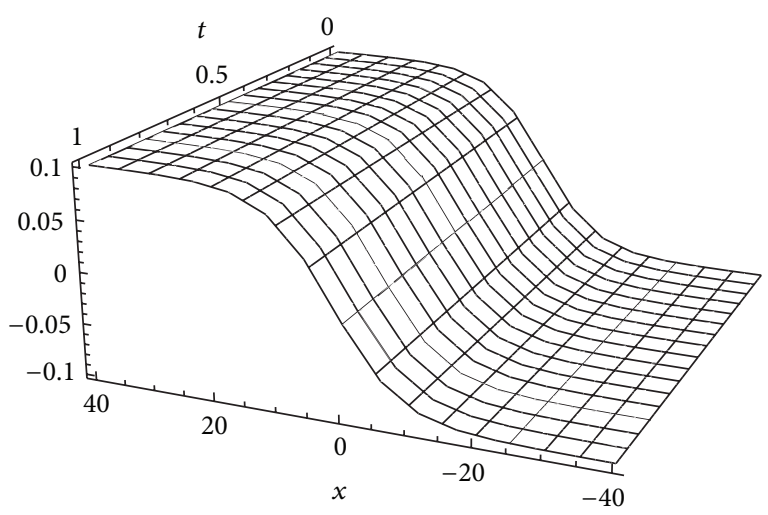

(a)

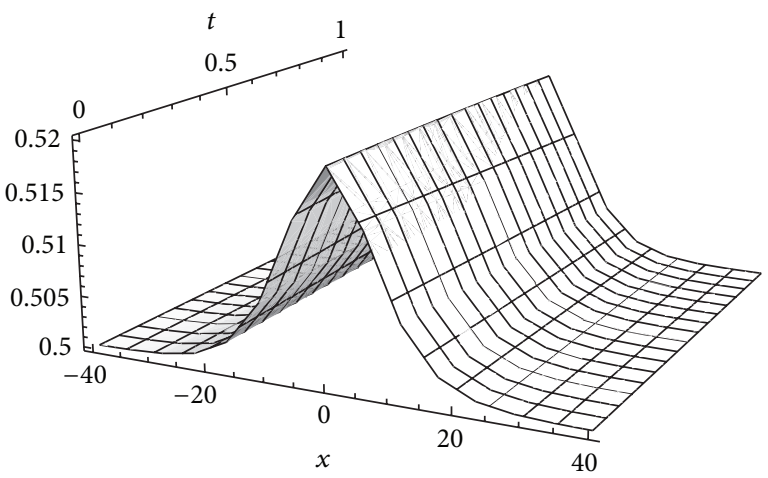

(b)

FIGURE 4: The surface shows the exact solutions (31): (a) $u(x, t)$; (b) $v(x, t)$ when $\lambda=1$ and $\gamma=0.1$.

$$
\begin{aligned}
& v_{3}(x, t)=\left\{2 \gamma^{5} \sec ^{8}(\gamma x)\right. \\
& \times[ 92 \gamma^{6}+3420 \gamma^{4} \lambda+351 \gamma^{2} \lambda^{2}+54 \lambda^{3} \\
&-9\left(12 \gamma^{6}+1456 \gamma^{4} \lambda+63 \gamma^{2} \lambda^{2}-9 \lambda^{3}\right) \\
& \times \sinh ^{2}(\gamma x) \\
&-96 \gamma^{2}\left(2 \gamma^{4}-48 \gamma^{2} \lambda+9 \lambda^{2}\right) \sinh ^{4}(\gamma x) \\
&\left.\left.+\left(2 \gamma^{2}-3 \lambda\right)^{3} \sinh (\gamma x)^{6}\right]\right\} \\
& \times \frac{\tanh (\gamma x) t^{3 \alpha}}{\Gamma(1+3 \alpha)} \\
&- {\left[27\left(\lambda-2 \gamma^{2}\right)^{2} \sinh (\gamma x)\right.} \\
&\left.-96 \gamma^{2}\left(2 \gamma^{2}-3 \lambda\right) \sinh ^{3}(\gamma x)\right] \\
& \times \frac{\gamma^{7} \sec ^{7}(\gamma x) \Gamma(1+2 \alpha) t^{3 \alpha}}{\Gamma(1+\alpha)^{2} \Gamma(1+3 \alpha)} .
\end{aligned}
$$

The effectiveness and accuracy of the approximate solutions can be seen from the comparison figures.

Figures 4 and 5 show the approximate solutions of (36) and (37) and the exact ones of (31) with $\alpha=\lambda=1$ and 
$\gamma=0.1$, respectively. Comparing Figures $1(\mathrm{a})$ and $1(\mathrm{~b})$ with Figures 2(a) and 2(b), we can see that the solutions obtained by different methods are nearly identical. From these figures, we can know that the series solutions converge rapidly, so a good approximation has been achieved.

\section{Summary and Discussion}

In this paper, combining the Caputo fractional derivative, the GDTM was applied to derive approximate analytical solutions of the time-fractional Hirota-Satsuma coupled KdV equation and coupled MKdV equation with initial conditions. The numerical solutions obtained from the GDTM are shown graphically. The obtained results demonstrate the reliability of the algorithm and its wider applicability to nonlinear fractional coupled partial differential equations.

In [8] and the references cited therein, the so-called fractional complex transform (FCT) is suggested to convert a fractional differential equation with Jumarie's modification of Riemann-Liouville derivative into its classical differential partner. According to the idea of FCT, for some fractional differential equations with Caputo time-fractional derivative $D_{t}^{\beta}$, we assume that

$$
\begin{aligned}
& u(x, t)=\sum_{m=0}^{+\infty} u_{m}(x) \frac{t^{m \beta}}{\Gamma(1+m \beta)}, \\
& v(x, t)=\sum_{m=0}^{+\infty} v_{m}(x) \frac{t^{m \beta}}{\Gamma(1+m \beta)},
\end{aligned}
$$

consequently, from Lemma 3,

$$
D_{t}^{\beta} u(x, t)=\sum_{m=0}^{+\infty} u_{m+1}(x) \frac{t^{m \beta}}{\Gamma(1+m \beta)},
$$

and it can be seen easily that

$$
\begin{aligned}
u(x, t) v(x, t)=\sum_{m=0}^{+\infty}\left(\sum_{r=0}^{m} u_{r}(x) v_{m-r}(x)\right. \\
\left.\quad \times \frac{\Gamma(1+m \beta)}{\Gamma(1+r \beta) \Gamma(1+(m-r) \beta)}\right) \\
\\
\times \frac{t^{m \beta}}{\Gamma(1+m \beta)} .
\end{aligned}
$$

We can point out that the approximate solutions of (11) and (29) by GDTM can be derived by the similar method compared with FCT. Without loss of generality, we only consider (29) with the initial conditions of (30). In fact, suppose that

$$
\begin{aligned}
& f(x, t)=\sum_{m=0}^{+\infty} f_{m}(x) \frac{t^{m \beta}}{\Gamma(1+m \beta)}, \\
& g(x, t)=\sum_{m=0}^{+\infty} g_{m}(x) \frac{t^{m \beta}}{\Gamma(1+m \beta)}, \\
& p(x, t)=\sum_{m=0}^{+\infty} p_{m}(x) \frac{t^{m \beta}}{\Gamma(1+m \beta)}, \\
& q(x, t)=\sum_{m=0}^{+\infty} q_{m}(x) \frac{t^{m \beta}}{\Gamma(1+m \beta)} .
\end{aligned}
$$

From (33), (40), and (41), we have the following iteration formulae:

$$
\begin{aligned}
& u_{0}(x)=u(x, 0), \quad v_{0}(x)=v(x, 0), \\
& f_{0}(x)=v_{0}^{\prime}(x), \quad g_{0}(x)=u_{0}^{\prime}(x), \\
& p_{0}(x)=g_{0}(x)+3 v_{0}(x), \\
& q_{0}(x)=u_{0}^{2}(x)-v_{0}(x)+\lambda, \\
& f_{m}(x)=v_{m}^{\prime}(x), \quad g_{m}(x)=u_{m}^{\prime}(x), \\
& p_{m}(x)=g_{m}(x)+3 v_{m}(x), \\
& q_{m}(x)=\sum_{r=0}^{m} u_{r}(x) u_{m-r}(x) \frac{\Gamma(1+m \beta)}{\Gamma(1+r \beta) \Gamma(1+(m-r) \beta)} \\
& -v_{m}(x), \\
& u_{m+1}(x)=\frac{1}{2} p_{m}^{\prime \prime}(x)-3 \sum_{r=0}^{m} q_{r}(x) g_{m-r}(x) \\
& \times \frac{\Gamma(1+m \beta)}{\Gamma(1+r \beta) \Gamma(1+(m-r) \beta)} \\
& +3 \sum_{r=0}^{m} u_{r}(x) f_{m-r}(x) \\
& \times \frac{\Gamma(1+m \beta)}{\Gamma(1+r \beta) \Gamma(1+(m-r) \beta)}, \\
& v_{m+1}(x)=-f_{m}^{\prime \prime}(x)-\sum_{r=0}^{m} g_{r}(x) p_{m-r}^{\prime}(x) \\
& \times \frac{\Gamma(1+m \beta)}{\Gamma(1+r \beta) \Gamma(1+(m-r) \beta)} \\
& +3 \sum_{r=0}^{m} q_{r}(x) f_{m-r}(x) \frac{\Gamma(1+m \beta)}{\Gamma(1+r \beta) \Gamma(1+(m-r) \beta)} \\
& +\sum_{r=0}^{m} g_{r}(x) g_{m-r}^{\prime}(x) \frac{\Gamma(1+m \beta)}{\Gamma(1+r \beta) \Gamma(1+(m-r) \beta)},
\end{aligned}
$$

for $m=0,1,2,3, \ldots$ 


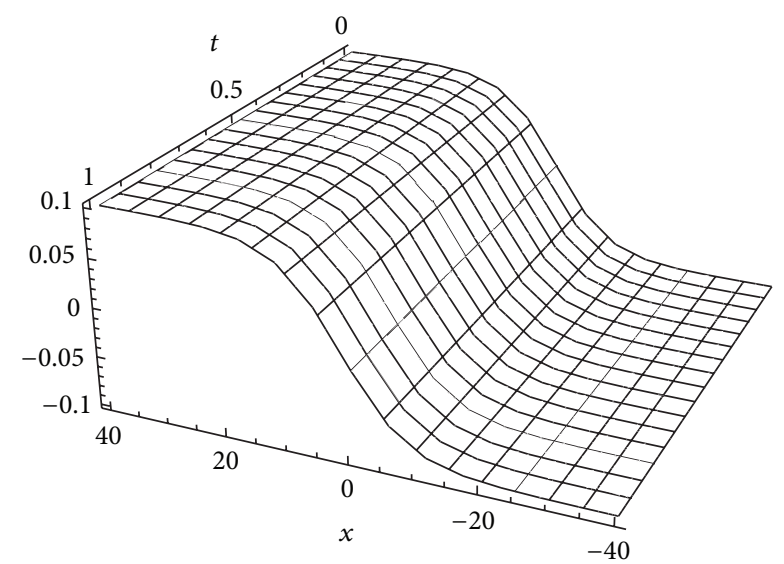

(a)

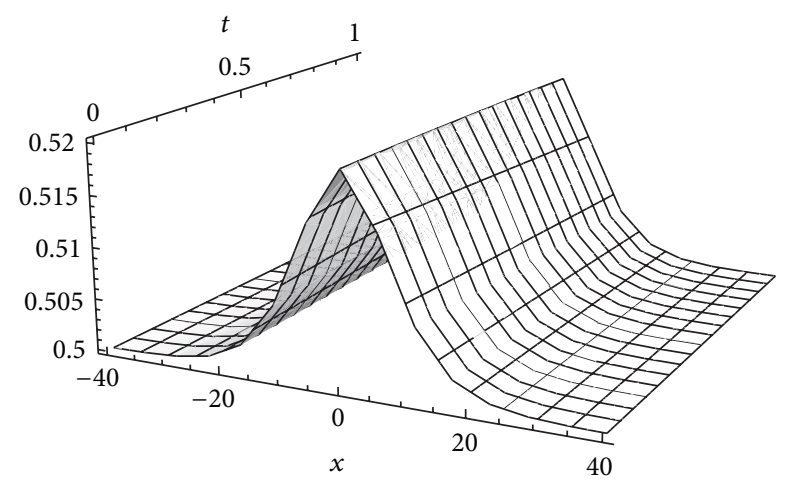

(b)

FIgURE 5: The surface shows the approximate solutions of (29): (a) $u(x, t)$ of (36); (b) $v(x, t)$ of (37) when $\alpha=\lambda=1$ and $\gamma=0.1$.

Setting $u_{m}(x, t)=u_{m}(x)\left(t^{m \beta} / \Gamma(1+m \beta)\right)$ and $v_{m}(x, t)=$ $v_{m}(x)\left(t^{m \beta} / \Gamma(1+m \beta)\right)$, and using (43), we can obtain the 3 order approximate solutions as (36) and (37) by using the symbol computational software Mathematica.

\section{Acknowledgments}

The project is supported by the NNSF of China (11061021), the NSF-IMU of China (2012MS0105, 2012MS0106), and the YSF-IMU of China (ND0811).

\section{References}

[1] S. Momani and Z. Odibat, "Homotopy perturbation method for nonlinear partial differential equations of fractional order," Physics Letters A, vol. 365, no. 5-6, pp. 345-350, 2007.

[2] C. Li and Y. Wang, "Numerical algorithm based on Adomian decomposition for fractional differential equations," Computers \& Mathematics with Applications, vol. 57, no. 10, pp. 1672-1681, 2009.

[3] S. R. Santanu, "Analytical solution for the space fractional diffusion equation by two-step Adomian decomposition method," Communications in Nonlinear Science and Numerical Simulation, vol. 14, no. 4, pp. 1295-1306, 2009.

[4] M. R. Yulita, M. S. M. Noorani, and I. Hashim, "Variational iteration method for fractional heat- and wave-like equations,"
Nonlinear Analysis. Real World Applications, vol. 10, no. 3, pp. 1854-1869, 2009.

[5] J. H. He, "Approximate analytical solution for seepage flow with fractional derivatives in porous media," Computer Methods in Applied Mechanics and Engineering, vol. 167, no. 1-2, pp. 57-68, 1998.

[6] H. Jafari and S. Seifi, "Solving a system of nonlinear fractional partial differential equations using homotopy analysis method," Communications in Nonlinear Science and Numerical Simulation, vol. 14, no. 5, pp. 1962-1969, 2009.

[7] H. Xu, S. J. Liao, and X.-C. You, "Analysis of nonlinear fractional partial differential equations with the homotopy analysis method," Communications in Nonlinear Science and Numerical Simulation, vol. 14, no. 4, pp. 1152-1156, 2009.

[8] J. H. He, S. K. Elagan, and Z. B. Li, "Geometrical explanation of the fractional complex transform and derivative chain rule for fractional calculus," Physics Letters A, vol. 376, no. 4, pp. 257259, 2012.

[9] Z. Odibat and S. Momani, "A generalized differential transform method for linear partial differential equations of fractional order," Applied Mathematics Letters, vol. 21, no. 2, pp. 194-199, 2008.

[10] J. H. He, "Approximate solution of nonlinear differnetial equations with convolution product nonlinearities," Computer Methods in Applied Mechanics and Engineering, vol. 167, pp. 6973, 1998.

[11] J. H. He, "Homotopy perturbation technique," Computer Methods in Applied Mechanics and Engineering, vol. 178, no. 3-4, pp. 257-262, 1999.

[12] G. Wu and E. W. M. Lee, "Fractional variational iteration method and its application," Physics Letters A, vol. 374, no. 25, pp. 2506-2509, 2010.

[13] G. Wu, "A fractional variational iteration method for solving fractional nonlinear differential equations," Computers \& Mathematics with Applications, vol. 61, no. 8, pp. 2186-2190, 2011.

[14] J. H. He, "A short remark on fractional variational iteration method," Physics Letters A, vol. 375, no. 38, pp. 3362-3364, 2011.

[15] J. Ji, J. Zhang, and Y. Dong, "The fractional variational iteration method improved with the Adomian series," Applied Mathematics Letters, vol. 25, no. 12, pp. 2223-2226, 2012.

[16] J. H. He and X.-H. Wu, "Variational iteration method: new development and applications," Computers \& Mathematics with Applications, vol. 54, no. 7-8, pp. 881-894, 2007.

[17] J. K. Zhou, Differential TransFormation and Its Applications for Electric CircuIts, Wuhan University Press, Wuhan, China, 1986, (in Chinese).

[18] A. S. V. Ravi Kanth and K. Aruna, "Solution of singular twopoint boundary value problems using differential transformation method," Physics Letters A, vol. 372, no. 26, pp. 4671-4673, 2008.

[19] A. Fatma, "Solutions of the system of differential equations by differential transform method," Applied Mathematics and Computation, vol. 147, no. 2, pp. 547-567, 2004.

[20] S. H. Chang and I. L. Chang, "A new algorithm for calculating one-dimensional differential transform of nonlinear functions," Applied Mathematics and Computation, vol. 195, no. 2, pp. 799805, 2008.

[21] S. H. Chang and I.-L. Chang, "A new algorithm for calculating two-dimensional differential transform of nonlinear functions," Applied Mathematics and Computation, vol. 215, no. 7, pp. 24862494, 2009. 
[22] K. C. Chen and S. H. Ho, "Solving partial differential equations by two-dimensional differential transform method," Applied Mathematics and Computation, vol. 106, no. 2-3, pp. 171-179, 1999.

[23] A. S. V. Ravi Kanth and K. Aruna, "Differential transform method for solving the linear and nonlinear Klein-Gordon equation," Computer Physics Communications, vol. 180, no. 5, pp. 708-711, 2009.

[24] S. Momani, Z. Odibat, and V. S. Erturk, "Generalized differential transform method for solving a space- and time-fractional diffusion-wave equation," Physics Letters A, vol. 370, no. 5-6, pp. 379-387, 2007.

[25] M. Kurulay and M. Bayram, "Approximate analytical solution for the fractional modified $\mathrm{KdV}$ by differential transform method," Communications in Nonlinear Science and Numerical Simulation, vol. 15, no. 7, pp. 1777-1782, 2010.

[26] E. G. Fan, "Soliton solutions for a generalized Hirota-Satsuma coupled KdV equation and a coupled MKdV equation," Physics Letters A, vol. 282, no. 1-2, pp. 18-22, 2001.

[27] I. Podlubny, Fractional Differential Equations, Academic Press, New York, NY, USA, 1999.

[28] M. Caputo, "Linear models of dissipation whose Q is almost frequency independent. Part II," A Journal of the Royal Statistical Society, vol. 13, pp. 529-539, 1967.

[29] M. Ishteva, R. Scherer, and L. Boyadjiev, "On the caputo operator of fractional calculus and c-Laguerrefunctions," http://www.cc.gatech.edu/ mishteva/paperMSRJ.pdf .

[30] S. Momani and Z. Odibat, "A novel method for nonlinear fractional partial differential equations: combination of DTM and generalized Taylor's formula," Journal of Computational and Applied Mathematics, vol. 220, no. 1-2, pp. 85-95, 2008. 


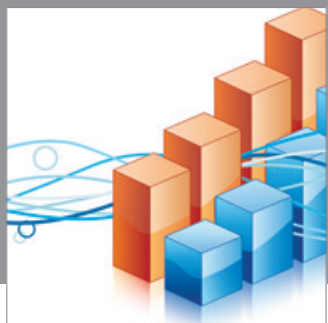

Advances in

Operations Research

mansans

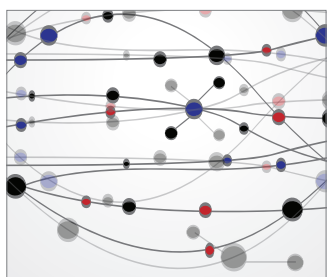

The Scientific World Journal
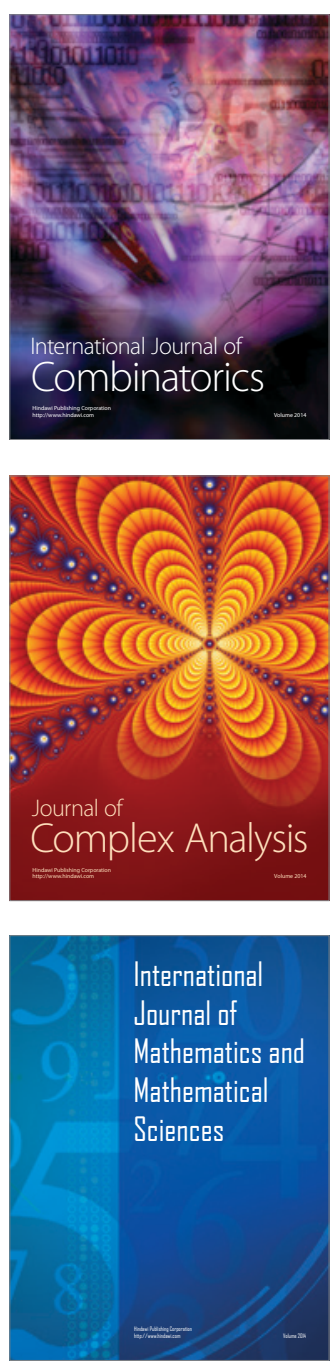
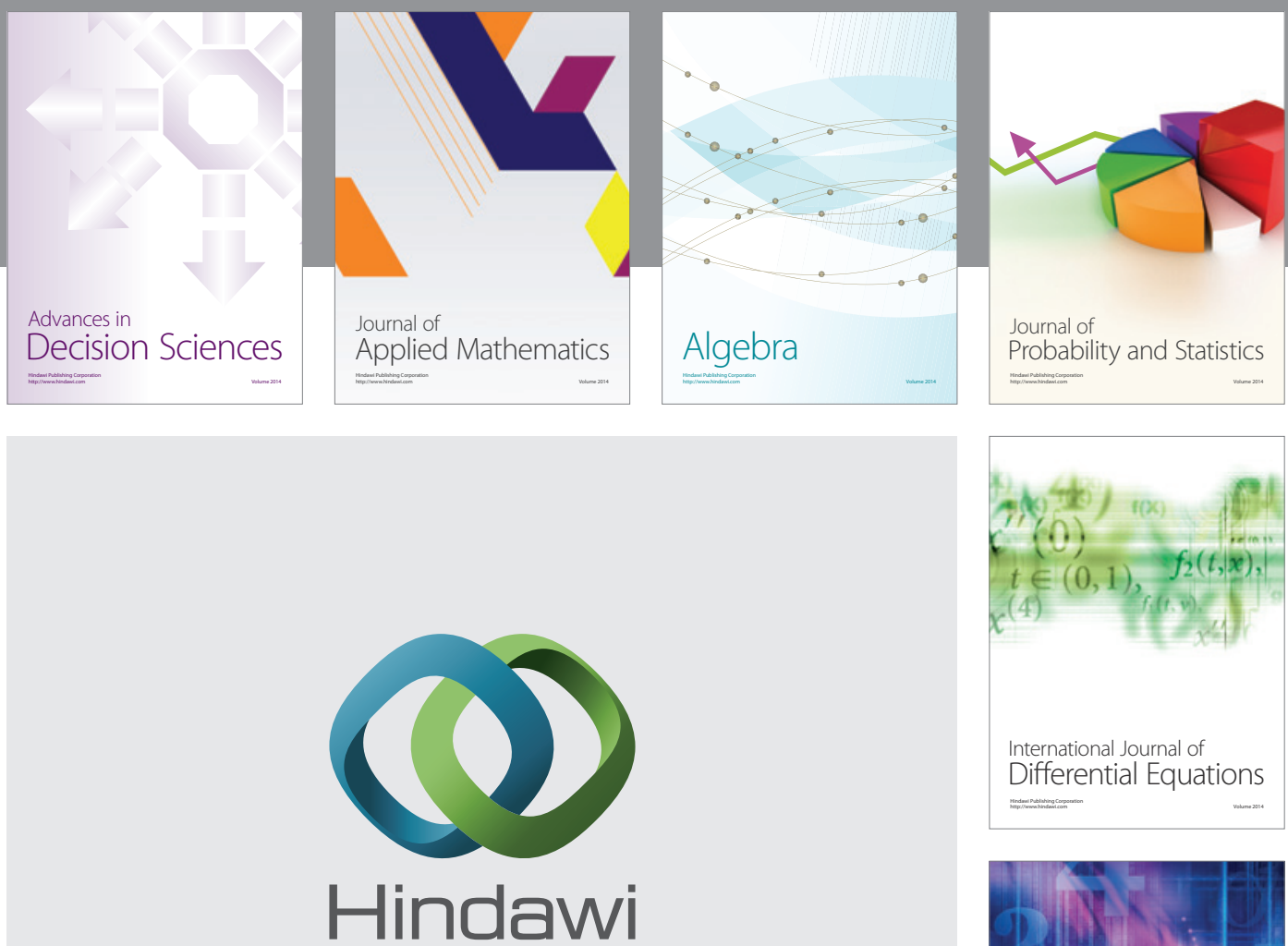

Submit your manuscripts at http://www.hindawi.com
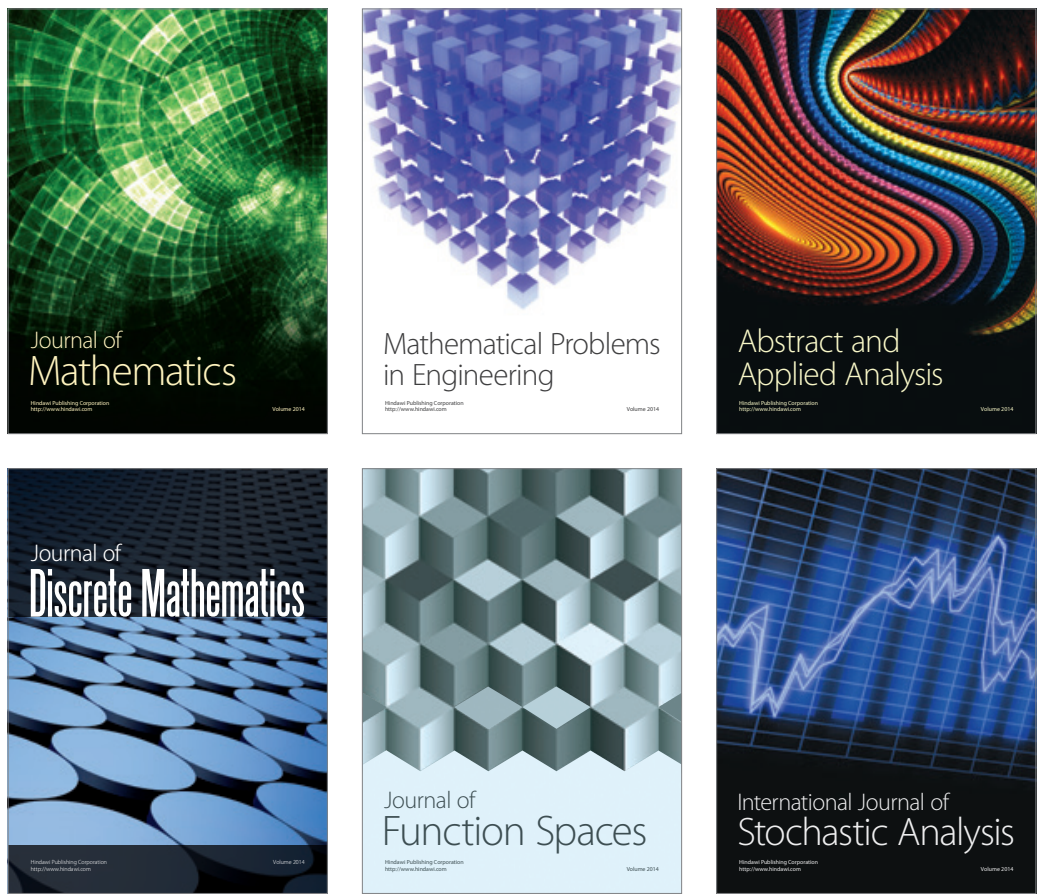

Journal of

Function Spaces

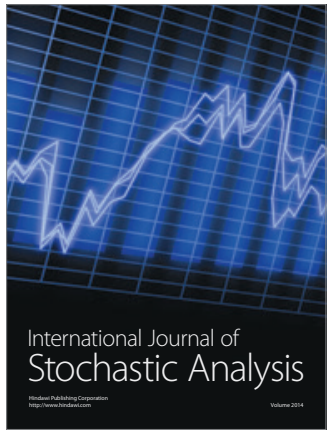

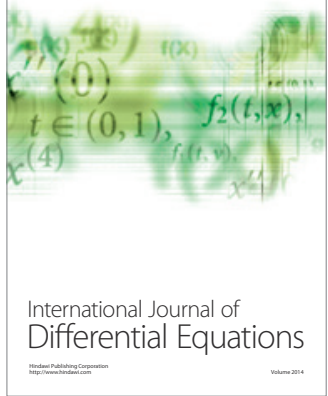
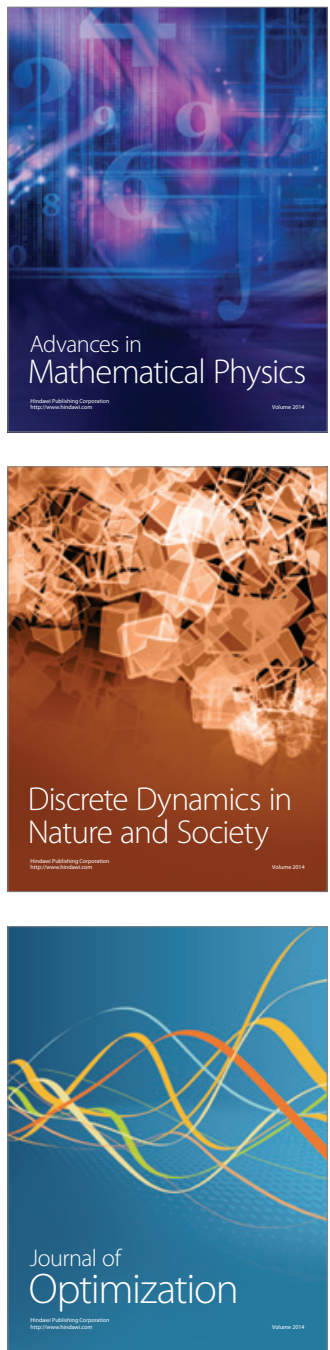